\title{
FACTORIZATION THEOREMS FOR MORPHISMS OF ORDERED GROUPOIDS AND INVERSE SEMIGROUPS
}

\author{
BENJAMIN STEINBERG \\ Faculdade de Ciências, da Universidade do Porto, \\ 4099-002 Porto, Portugal (bsteinbg@agc0.fc.up.pt)
}

(Received 15 September 1999)

\begin{abstract}
Adapting the theory of the derived category to ordered groupoids, we prove that every ordered functor (and thus every inverse and regular semigroup homomorphism) factors as an enlargement followed by an ordered fibration. As an application, we obtain Lawson's version of Ehresmann's Maximum Enlargement Theorem, from which can be deduced the classical theory of idempotent-pure inverse semigroup homomorphisms and E-unitary inverse semigroups.
\end{abstract}

Keywords: ordered groupoids; groupoids; semidirect products; inverse semigroups; fibrations; derived categories

AMS 2000 Mathematics subject classification: Primary 20M18; 20L05; 20M17

\section{Introduction}

In a series of several papers $[\mathbf{7}, \mathbf{8}, \mathbf{1 0}]$, as well as in his book $[\mathbf{1 1}]$, Lawson argues persuasively of the existence of an important connection between the study of inverse semigroups and that of ordered groupoids. He then proves, with an entirely coordinate-free approach, the McAlister $P$-theorem [12], and redevelops O'Carroll's theory of idempotent-pure homomorphisms of inverse semigroups $[\mathbf{1 6}, \mathbf{1 7}]$ using the techniques of ordered groupoids. The key to Lawson's approach is Ehresmann's Maximum Enlargement Theorem [11, Theorem 8.3.3], characterizing star-injective morphisms of ordered groupoids.

This paper associates to each ordered functor $\varphi$ an ordered groupoid $\operatorname{Der}(\varphi)$ called its derived ordered groupoid; such an approach for monoids goes back to [21]. Using the derived ordered groupoid and the semidirect product, we are able to show that every ordered groupoid morphism factors as an enlargement followed by an ordered fibration. This means, intuitively, that up to Morita equivalence every ordered functor is a fibration (surjective on $\mathcal{R}$-classes in semigroup terms).

We then show that a broad class of ordered functors has the property of having a 'best-possible' factorization through a covering morphism; it is from this result, in the star-injective case, that we deduce the Maximum Enlargement Theorem.

In terms of inverse semigroup theory, some of our results can be interpreted as follows. 
(i) Let $\varphi: S \rightarrow T$ be an idempotent-pure (pre)-homomorphism. Then the partially ordered set in the McAlister-O'Carroll representation is the set of $\mathcal{D}$-classes of $\operatorname{Der}(\varphi)$ ordered by the $\mathcal{J}$-order.

(ii) If $\equiv$ is a congruence on an inverse semigroup $S$ such that each idempotent equivalence class is a semigroup with $\mathcal{J}=\mathcal{D}$, then there is a minimal congruence $\sim \subseteq \equiv$ such that the natural map $S / \sim \rightarrow S / \equiv$ is idempotent pure. This always occurs, in particular, if $S$ is semisimple.

(iii) If $\equiv$ is a congruence on an inverse semigroup $S$ such that each idempotent equivalence class is a Clifford semigroup, then the congruence $\sim$ described above exists and is idempotent separating.

(iv) Our paper offers a natural definition of the semidirect product of inverse semigroups; this will be investigated further in future work.

Our work can be viewed as a generalization of the approach of Margolis and Pin [14] to the $P$-theorem. Another such generalization was made by Gomes and Szendrei [3]. In fact, hitherto unpublished work by the author, Lawson and Margolis shows that their approach is related to ours via what is called in [11] the pseudoproduct; more concretely, their derived quiver of a morphism of inverse semigroups is the resulting algebraic structure obtained from applying the pseudoproduct to our derived ordered groupoid of the corresponding morphism of ordered groupoids.

This work grew out of the work of the author and Tilson on derived categories [20].

\section{Preliminaries and notation}

In this paper morphisms will generally be written on the right of their arguments. The domain and range of arrows of a category will be defined accordingly.

A category $C$ is a set equipped with a partial binary operation, which we shall denote by concatenation, satisfying several axioms which we shall list below. We will write $\exists x y$ if the product $x y$ exists. An element $e$ of $C$ will be called an identity if $\exists e x$ implies $e x=x$ and $\exists x e$ implies $x e=x$. We use $C_{\mathrm{o}}$ for the set of identities (or objects) of $C$. Elements of $C$ will often be called arrows. The axioms for a category are the following:

(C1) $\exists x(y z)$ if and only $\exists(x y) z$ in which case they are equal;

(C2) $\exists x(y z)$ if and only if $\exists x y$ and $\exists y z$; and

(C3) for each $x \in C$, there exists identities $e, f$ such that $\exists x e$ and $\exists f x$.

One can show that the identities in axiom (C3) are uniquely determined by $x$. We let $x \boldsymbol{d}=f$ and $x \boldsymbol{r}=e ; x \boldsymbol{d}$ is called the domain of $x$ and $x \boldsymbol{r}$ is called the range. Note that $\exists x y$ if and only if $x \boldsymbol{r}=y \boldsymbol{d}$. Conversely, if there are functions $\boldsymbol{d}, \boldsymbol{r}: C \rightarrow C_{\mathrm{o}}$ such that $\exists x y$ if and only if $x \boldsymbol{r}=y \boldsymbol{d}, x \boldsymbol{d} x=x=x x \boldsymbol{r}$, and $x(y z)=(x y) z$ when both sides exist, then the above axioms are satisfied. Observe that $C_{\mathrm{o}}$ is a subcategory of $C$. In truth, however, we will only use this definition of categories when we view them as algebraic 
objects. When we use categories as classifying objects, then we will stick to the more classical formulation in terms of object and arrow sets. A subcategory $D$ of a category $C$ is called a full subcategory if it contains all arrows of $C$ between its objects. We use $C^{\text {op }}$ for $C$ with the reverse multiplication.

A groupoid is a category $G$ such that, for each $x \in G$, there is an element $x^{-1} \in X$ such that $x \boldsymbol{d}=x^{-1} \boldsymbol{r}, x \boldsymbol{r}=x^{-1} \boldsymbol{d}, x x^{-1}=x \boldsymbol{d}$, and $x^{-1} x=x \boldsymbol{r}$. We leave it to the reader to check that $x^{-1}$ is uniquely determined by $x$. A groupoid $G$ is said to be connected, if for each $e, f \in G_{\mathrm{o}}$ there is an element $x$ with $x \boldsymbol{d}=e, x \boldsymbol{r}=f$. The maximal connected subgroupoids of a groupoid are called the connected components of $G$. In analogy to semigroup theory, if arrows $g, g^{\prime} \in G$ are in the same connected component, we write $g \mathcal{D} g^{\prime}$ and use the term $\mathcal{D}$-class as a synonym for connected component. We will denote the $\mathcal{D}$-class of $g$ by $D_{g}$.

If $C, D$ are categories, then $\varphi: C \rightarrow D$ is a morphism or functor if $\exists x y$ implies that $(x y) \varphi=x \varphi y \varphi$ and for all $x, x \boldsymbol{d} \varphi=x \varphi \boldsymbol{d}, x \boldsymbol{r} \varphi=x \varphi \boldsymbol{r}$. Note that if $G$ and $H$ are groupoids and $\varphi: G \rightarrow H$ is such that $\exists x y$ implies that $(x y) \varphi=x \varphi y \varphi$, then $\varphi$ is a morphism and $x^{-1} \varphi=(x \varphi)^{-1}$.

Suppose $G$ is a groupoid and $\leqslant$ is a partial order on $G$. Then $G$ is called an ordered groupoid if the following axioms hold.

(O1) $x \leqslant y \Longrightarrow x^{-1} \leqslant y^{-1}$.

(O2) $x \leqslant y, u \leqslant v, \exists x u, \exists y v \Longrightarrow x u \leqslant y v$.

(O3) If $x \in G$ and $e \leqslant x \boldsymbol{d}$, then there exists a unique element $(e \mid x)$, called the restriction of $x$ to $e$, such that $(e \mid x) \boldsymbol{d}=e$ and $(e \mid x) \leqslant x$.

(O4) If $x \in G, e \in G_{\mathrm{o}}$ and $e \leqslant x \boldsymbol{r}$, then there exists a unique element $(x \mid e)$, called the corestriction of $x$ to $e$, such that $(x \mid e) \boldsymbol{r}=e$ and $(x \mid e) \leqslant x$.

It can be shown $[\mathbf{1 1}]$ that $(\mathrm{O} 4)$ is implied by the other axioms.

A subset $X$ of a partially ordered set $Y$ is called an order ideal if $y \leqslant x \in X$ implies that $y \in X$. It can be shown $[\mathbf{1 1}]$ that $(G, \leqslant)$ is an ordered groupoid if and only if (O1) and (O2) hold, $G_{\mathrm{O}}$ is an order ideal, and that, for $e \in G_{\mathrm{O}}, e \leqslant x \in G$ implies that there exists $y \leqslant x$ with $y \boldsymbol{d}=e$. We will frequently use this fact in the sequel. Any groupoid is ordered with respect to the equality relation, and when we say groupoid without further qualification we mean a groupoid ordered by equality.

A partially ordered set $X$ is called a meet semilattice if, for every $x, y \in X$, there is a greatest lower bound $x \wedge y$. In this case, $X$ becomes a semigroup under the meet operation. An ordered groupoid $G$ is called an inductive groupoid if $G_{\mathrm{O}}$ is a meet semilattice. Any group is an inductive groupoid. An inverse semigroup can be made into an inductive groupoid by viewing its $\mathcal{D}$-classes as groupoids (with the restricted or trace product) and using the natural partial order. Conversely, any inductive groupoid can be viewed as an inverse semigroup (see [11] for more).

If $G$ is an ordered groupoid, and $g, g^{\prime} \in G$, we write $g \leqslant \mathcal{J} g^{\prime}$ if there exists $h \in G$ such that $g \mathcal{D} h \leqslant g^{\prime}$. One can easily verify that this is a preorder. We use $\mathcal{J}$ to denote 
the associated equivalence relation. This notation comes by analogy to semigroup theory (cf. [11, Proposition 3.2.8]). Observe that there is a containment $\mathcal{D} \subseteq \mathcal{J}$ whence $\leqslant \mathcal{J}$ induces a preorder on the set of $\mathcal{D}$-class of $G$, which we will also denote by $\leqslant \mathcal{J}$. This preorder is given by

$$
D_{1} \leqslant \mathcal{J} D_{2} \Longleftrightarrow \forall h \in D_{2}, \exists g \in D_{1} \quad \text { such that } g \leqslant h,
$$

where $D_{1}$ and $D_{2}$ are $\mathcal{D}$-classes of $G$.

An ordered functor is a functor $\varphi: G \rightarrow H$ such that $x \leqslant y \Longrightarrow x \varphi \leqslant y \varphi$. An inductive functor is an ordered functor between inductive groupoids which preserves the meet of identities. An ordered functor $\varphi: G \rightarrow H$ is called an order embedding if $x \varphi \leqslant y \varphi$ if and only if $x \leqslant y$. In such a situation, $G$ can be viewed as an ordered subgroupoid of $H$. One can show [11] that, for an ordered functor $\varphi,(e \mid g) \varphi=(e \varphi \mid g \varphi)$ and that $(g \mid f) \varphi=(g \varphi \mid f \varphi)$.

If $\left\{X_{j}\right\}_{j \in I}$ is a collection of partially ordered sets, then $\prod_{j \in I} X_{j}$ is a partially ordered set, where we define $\left(x_{j}\right)_{j \in I} \leqslant\left(y_{j}\right)_{j \in I}$ if $x_{j} \leqslant y_{j}$ for all $j \in I$. This order is called the product order. If $\left\{G_{j}\right\}_{j \in I}$ is a collection of groupoids, then $\prod G_{j}$ is a groupoid under coordinatewise multiplication. If the $G_{j}$ are ordered or inductive groupoids, so is $\prod G_{j}$ under the product order. This product has the standard universal property of products.

If $\varphi: X \rightarrow Y$ and $\psi: Z \rightarrow Y$ are morphisms, the pullback is $X \times_{\varphi, \psi} Z=\{(x, z) \mid x \varphi=$ $z \psi\}$. It is not hard to verify that if $\varphi: G \rightarrow H$ and $\psi: G^{\prime} \rightarrow H$ are morphisms of ordered (inductive) groupoids, then $G \times{ }_{\varphi, \psi} G^{\prime}$ is an ordered (inductive) subgroupoid of $G \times G^{\prime}$ with the standard universal property of a pullback. The only non-trivial verification is that if $(e, f) \leqslant\left(g, g^{\prime}\right) \boldsymbol{d} \in G \times \times_{\varphi, \psi} G^{\prime}$, then

$$
(e \mid g) \varphi=(e \varphi \mid g \varphi)=\left(f \psi \mid g^{\prime} \psi\right)=\left(f \mid g^{\prime}\right) \psi
$$

and so $\left(e|g, f| g^{\prime}\right) \in G \times \times_{\varphi, \psi} G^{\prime}$. Also, we note that if $G, G^{\prime}$ and $H$ are inductive groupoids and $\varphi, \psi$ preserve the meet, then the pullback is an inductive groupoid.

We use OGrp for the category of ordered groupoids and order-preserving morphisms, while we use Ind for the category of inductive groupoids and meet-preserving morphisms. Of course, Ind is a subcategory of OGrp. It follows from the existence of pullbacks and arbitrary products, that these categories are complete, that is, they have all limits.

The reader is referred to [11, Chapter 4] for basic results on ordered groupoids; we caution the reader that his definitions of $\boldsymbol{d}$ and $\boldsymbol{r}$ are reversed from ours.

We use Morph to denote the category of ordered functors. That is, the objects of Morph are ordered functors $\varphi: G \rightarrow H$, and an arrow between $\varphi$ and $\psi$ is a pair $(\tau, \rho)$ of ordered functors making a commuting square

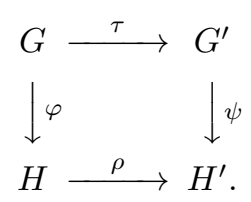

We use $\mathbf{M o r p h}_{\mathrm{I}}$ for the subcategory whose objects are inductive functors and whose arrows are ordered pairs of inductive functors making the appropriate diagram commute. 
If $X$ is a partially ordered set, we can make $X$ into a 'discrete ordered groupoid' $X_{\mathrm{D}}$ by defining a partial multiplication $\exists x y$ if and only if $x=y$, in which case $x x=x$. Note that if $X$ is a meet semilattice, then $X_{\mathrm{D}}$ is an inductive groupoid. Observe that $\left(X_{\mathrm{D}}\right)_{\mathrm{o}}=X_{\mathrm{D}}$, whence the name discrete. If $H$ is a groupoid, $H$ can be viewed in a natural way as a partially ordered set by forgetting the multiplication; we then use $H_{\mathrm{D}}$ to denote the discrete ordered groupoid obtained from $H$ viewed as a partially ordered set.

\section{The adjunction}

In this section we define the semidirect product of ordered groupoids and the derived ordered groupoid of an ordered functor. As in the case of categories [20], these will be functors standing in an adjoint relation (see [13] for the relevant definitions). The universal property of the derived ordered groupoid will then be used to prove our various factorization results. This adjunction, in the category setting, is due to Nico [15] and Kientzle [6].

\subsection{Ordered groupoid actions}

We now define what it means for one ordered (inductive) groupoid to act on another. This will then allow us to define a semidirect product of ordered (inductive) groupoids. Our definition on the one hand is a generalization of the notion for groupoids of Brown [1] and Houghton [5] (which, of course, generalizes the usual notion for groups), and on the other it is a generalization of Lawson's notion of an ordered representation [11]; see these papers for examples (examples in the category setting can be found in [20]).

Let $G$ and $H$ be ordered groupoids. Then a left action $(\pi, A)$ of $H$ on $G$ consists of the following data: an ordered functor $\pi: G \rightarrow H_{\mathrm{o}}$ and an ordered functor $A: H_{\mathrm{D}} \times_{\boldsymbol{r}, \pi} G \rightarrow G$ (which we normally denote by left exponentiation, $(h, g) A={ }^{h} g$ ) such that the following axioms hold (we use $\exists^{h} g$ if $h \boldsymbol{r}=g \pi$ ).

(A1) If $\exists^{h} g$, then $\left({ }^{h} g\right) \pi=h \boldsymbol{d}$.

(A2) If $\exists h_{1} h_{2}, \exists^{h_{2}} g$, then ${ }^{h_{1}}\left({ }^{h_{2}} g\right)={ }^{h_{1} h_{2}} g$.

(A3) ${ }^{g \pi} g=g$.

Note that axiom (A1) implies that in (A2), $\exists^{h_{1}}\left({ }^{h_{2}} g\right)$. If $G$ and $H$ are inductive groupoids, then the left action is called inductive if $\pi$ is a morphism of inductive groupoids.

We now collect several of the most important properties of an action into a proposition which shall be used throughout this paper without comment.

Proposition 3.1. Let $G, H$ be ordered groupoids and suppose $H$ acts on the left of $G$ via $(\pi, A)$.

(1) If $h \in H, g \in G$, and $\exists^{h} g$, then ${ }^{h^{-1}}\left({ }^{h} g\right)=g$.

(2) If $g, g^{\prime} \in G$ and $\exists g g^{\prime}$, then $\pi g=\pi g^{\prime}=\pi\left(g g^{\prime}\right)$.

(3) If $g \leqslant g^{\prime} \in G, h \leqslant h^{\prime} \in H$ and $\exists^{h} g, \exists^{h^{\prime}} g^{\prime}$, then ${ }^{h} g \leqslant h^{h^{\prime}} g^{\prime}$. 
(4) If $g, g^{\prime} \in G, h \in H$ and $\exists^{h} g, \exists g g^{\prime}$, then $\exists^{h}\left(g g^{\prime}\right), \exists^{h} g^{\prime}$, and ${ }^{h}\left(g g^{\prime}\right)={ }^{h} g^{h} g^{\prime}$.

(5) If $e \in G_{\mathrm{o}}, h \in H$, and $\exists^{h} e$, then ${ }^{h} e \in G_{\mathrm{o}} \cap(h \boldsymbol{d}) \pi^{-1}$.

(6) For $e \in H_{\mathrm{o}}, e \pi^{-1}$ is a full ordered subgroupoid of $G$ and, for $h \in H$, left translation by $h$ induces an ordered isomorphism from $(h \boldsymbol{r}) \pi^{-1}$ to $(h \boldsymbol{d}) \pi^{-1}$. Furthermore, the association taking an arrow of $H$ to this ordered isomorphism induces a functor from $H^{\mathrm{op}}$ to OGrp.

(7) If $h \in H, g \in G$ and $\exists^{h} g$, then $\exists^{h}\left(g^{-1}\right)$ and ${ }^{h}\left(g^{-1}\right)=\left({ }^{h} g\right)^{-1}$.

(8) If $G$ and $H$ are inductive groupoids and the action is inductive, then the association of (6) is a functor from $H^{\text {op }}$ to Ind.

\section{Proof.}

(1) Since $\exists^{h} g, h^{-1} h=h \boldsymbol{r}=g \pi$. By (A2) ${ }^{h^{-1}}\left({ }^{h} g\right)={ }^{h^{-1} h} g={ }^{g \pi} g=g$ by (A3).

(2) This is clear since $\pi$ is a functor and $H_{\mathrm{o}}$ is discrete.

(3) We have $(h, g),\left(h^{\prime}, g^{\prime}\right) \in H_{\mathrm{D}} \times_{\boldsymbol{r}, \pi} G$ and $(h, g) \leqslant\left(h^{\prime}, g^{\prime}\right)$. The result follows since $A$ preserves order.

(4) By property (2) above, $\exists^{h}\left(g g^{\prime}\right), \exists^{h} g^{\prime}$. So, in $H_{\mathrm{D}} \times_{\boldsymbol{r}, \pi} G$, we can find $(h, g),\left(h, g^{\prime}\right)$, $\left(h, g g^{\prime}\right)$. But $(h, g)\left(h, g^{\prime}\right)=\left(h, g g^{\prime}\right)$ so the result follows since $A$ is a morphism.

(5) It follows that $(h, e) \in\left(H_{\mathrm{D}} \times_{\boldsymbol{r}, \pi} G\right)_{\mathrm{o}}$ and so $(h, e) A={ }^{h} e$ is an identity. We can deduce from (A1) that ${ }^{h} e$ is in $(h \boldsymbol{d}) \pi^{-1}$.

(6) This follows easily from the above properties and (A1), (A2) and (A3).

(7) Follows straight from property (6), since ordered functors preserve the inverse operation.

(8) Since $\pi$ is an inductive functor, if $e, f \in G_{\mathrm{o}}$ with $e \pi=f \pi,(e \wedge f) \pi=e \pi \wedge f \pi=e \pi$. Suppose $h \in H$ and $e, f \in G_{\mathrm{o}} \cap(h \boldsymbol{r}) \pi^{-1}$. By property (3), ${ }^{h}(e \wedge f) \leqslant{ }^{h} e,{ }^{h} f$, and so ${ }^{h}(e \wedge f) \leqslant{ }^{h} e \wedge{ }^{h} f$. But applying the same reasoning to $h^{-1}$, we see, by property (1), that $h^{-1}\left({ }^{h} e \wedge{ }^{h} h\right) \leqslant e \wedge f$ and hence, again using properties (1) and (3), we see that ${ }^{h} e \wedge{ }^{f} h={ }^{h}(e \wedge f)$.

Property (6) gives a 'geometric viewpoint' on what is an action. Essentially, one has a bundle $G$ of ordered groupoids lying over $H_{\mathrm{o}}$, where the map $\pi$ is the bundle projection map. Arrows of $H$ then induce ordered isomorphisms of the fibres. However, the bundle picture fails to capture that $G$ is ordered globally and that $\pi$ relates this global ordering to the ordering of $H_{\mathrm{o}}$.

The following example of an action will be of use later in the paper. 
Example 3.2. Let $H$ be an ordered (inductive) groupoid. Then $H$ acts (inductively) on $H_{\mathrm{o}}$ via $1_{H}: H_{\mathrm{o}} \rightarrow H_{\mathrm{o}}$, the identity, and ${ }^{h} h \boldsymbol{r}=h \boldsymbol{d}$.

We define a category Act whose objects consist of triples $(G, H,(\pi, A))$ where $H$ acts on $G$ via $(\pi, A)$. Usually we suppress $(\pi, A)$, just writing ${ }_{H} G$ to denote an ordered groupoid $G$ with a left action by $H$. An arrow from ${ }_{H} G$ to ${ }_{H^{\prime}} G^{\prime}$ consists of a pair of ordered functors $(\psi, \rho), \psi: G \rightarrow G^{\prime}$ and $\rho: H \rightarrow H^{\prime}$, which respect the action; that is, $g \pi_{H} \rho=g \psi \pi_{H^{\prime}}$ (where $\pi_{H}, \pi_{H^{\prime}}$ are the bundle projections for $G$ and $G^{\prime}$, respectively) and $\exists^{h} g$ implies that $\left({ }^{h} g\right) \psi={ }^{h \rho} g \psi$. Note that the first condition implies that if $\exists^{h} g$, then $g \psi \pi_{H^{\prime}}=g \pi_{H} \rho=(h \boldsymbol{r}) \rho$ so $\exists^{h \rho} g \psi$. We use $\mathbf{A c t}_{\mathrm{I}}$ to denote the subcategory where all the groupoids, actions and ordered functors are inductive.

\subsection{The semidirect product functor}

Given a left action of an ordered groupoid $H$ on an ordered groupoid $G$, we now define the semidirect product by $G \rtimes H$; observe that we are again suppressing the action in our notation. This construction, in the unordered setting, is what is called by Ehresmann [2] the crossed product, and by most category theorists the Grothendieck construction [4]. Examples include the usual semidirect product of groups; for more examples, see $[\mathbf{1}, \mathbf{5}]$ for the unordered case and [11] for the case of an ordered groupoid acting on a partially ordered set; further examples of the Grothendeick construction, viewed as a semidirect product, can be found in $[\mathbf{2 0}]$.

As a partially ordered set, $G \rtimes H=G \times_{\pi, \boldsymbol{d}} H$. Note that if $G$ and $H$ are finite, so is $G \rtimes H$. To define a product, first note that if $(g, h),\left(g^{\prime}, h^{\prime}\right) \in G \rtimes H$ and $\exists h h^{\prime}$, then $g^{\prime} \pi=h^{\prime} \boldsymbol{d}=h \boldsymbol{r}$ so ${ }^{h} g^{\prime}$ is defined. Then $\exists(g, h)\left(g^{\prime}, h^{\prime}\right)$ if $\exists h h^{\prime}$ and $\exists g^{h} g^{\prime}$, in which case we define

$$
(g, h)\left(g^{\prime}, h^{\prime}\right)=\left(g^{h} g^{\prime}, h h^{\prime}\right) .
$$

Proposition 3.3. One has that $G \rtimes H$ is an ordered groupoid. If $G, H$ and the action are all inductive, then $G \rtimes H$ is an inductive groupoid.

Proof. It is straightforward to see that

$$
(G \rtimes H)_{\mathrm{o}}=G_{\mathrm{o}} \times_{\pi, 1_{H_{\mathrm{o}}}} H_{\mathrm{o}},
$$

$(g, h) \boldsymbol{d}=(g \boldsymbol{d}, h \boldsymbol{d})$, and $(g, h) \boldsymbol{r}=\left({ }^{h^{-1}} g \boldsymbol{r}, h \boldsymbol{r}\right)$. It is then routine to verify associativity and so $G \rtimes H$ is a category. In fact, it is a groupoid: $(g, h)^{-1}=\left(h^{-1} g^{-1}, h^{-1}\right)$. The reader is referred to $[\mathbf{5}, \mathbf{2 0}]$ for details.

To verify (O1), note that if $(g, h) \leqslant(r, s)$, then $h^{-1} \leqslant s^{-1}, g \leqslant r$ and $h^{-1} g^{-1} \leqslant s^{-1} r^{-1}$. So $(g, h)^{-1}=\left({ }^{h^{-1}} g^{-1}, h^{-1}\right) \leqslant\left({ }^{-1} r^{-1}, s^{-1}\right)=(r, s)^{-1}$.

As for $(\mathrm{O} 2)$, if

$$
(g, h) \leqslant(r, s),(j, k) \leqslant(u, v)
$$

and

$$
\exists(g, h)(j, k), \exists(r, s)(u, v),
$$

then $h k \leqslant s v$ and ${ }^{h} j \leqslant{ }^{s} u$. So $(g, h)(j, k)=\left(g^{h} j, h k\right) \leqslant\left(r^{s} u, s v\right)=(r, s)(u, v)$. 
To see that $(G \rtimes H)_{\mathrm{o}}=G_{\mathrm{O}} \times_{\pi, 1_{H_{\mathrm{o}}}} H_{\mathrm{o}}$ is an order ideal, note that if $(g, h) \leqslant(e, f) \in$ $(G \rtimes H)_{\mathrm{o}}$, then $g \leqslant e, h \leqslant f$ implies that

$$
(g, h) \in G_{\mathrm{O}} \times_{\pi, 1_{H_{\mathrm{o}}}} H_{\mathrm{o}}=(G \rtimes H)_{\mathrm{o}} .
$$

Suppose $(e, f) \leqslant(g, h) \boldsymbol{d}=(g \boldsymbol{d}, h \boldsymbol{d})$. Then $e \leqslant g \boldsymbol{d}$ and $f \leqslant h \boldsymbol{d}$, so there exist $(e \mid g)$ and $(f \mid h)$. Since $(e \mid g) \pi=e \pi=f=(f \mid h) \boldsymbol{d},((e \mid g),(f \mid h)) \in G \rtimes H$. But $((e \mid g),(f \mid h)) \boldsymbol{d}=(e, f)$ and $((e \mid g),(f \mid h)) \leqslant(g, h)$.

If $G$ and $H$ are inductive, and the action is as well, then we claim $(e, f) \wedge\left(e^{\prime}, f^{\prime}\right)=$ $\left(e \wedge e^{\prime}, f \wedge f^{\prime}\right)$. Indeed, since $\pi$ preserves meets, $(G \rtimes H)_{\mathrm{o}}=G_{\mathrm{O}} \times_{\varphi, 1_{H_{\mathrm{O}}}} H_{\mathrm{O}}$ is a meet semilattice under pointwise meet.

Observe that the projection $\psi: G \rtimes H \rightarrow H$ defined by $(g, h) \psi=h$ is clearly a morphism. In the inductive case, the projection is meet preserving. Thus $\psi \in$ Morph $\left(\mathbf{M o r p h}_{\mathrm{I}}\right)$. We claim that the correspondence $\mathbf{S d}$ given by $\mathbf{S d}\left({ }_{H} G\right)=\psi: G \rtimes H \rightarrow H$ is a functor.

Proposition 3.4. The correspondence Sd : Act $\rightarrow$ Morph is a functor called the semidirect product functor. The restriction of this functor to $\mathbf{A c t}_{\mathrm{I}}$ has codomain $\operatorname{Morph}_{\mathrm{I}}$.

Proof. If $(\psi, \rho):{ }_{H} G \rightarrow{ }_{H^{\prime}} G^{\prime}$, then it is a simple verification to see that $\psi \times \rho:$ $G \rtimes H \rightarrow G^{\prime} \rtimes H^{\prime}$ is an ordered functor and that $(\psi \times \rho, \rho)$ gives a morphism of the projections. It is then immediate that $\mathbf{S d}$ is a functor.

If $(\psi, \rho)$ is an arrow of Act, we will use $(\psi \rtimes \rho, \rho)$ to denote the arrow of Morph obtained by applying the semidirect product functor.

Example 3.5. If we let $H$ act on $H_{\mathrm{o}}$ as in Example 3.2, then it is easy to see that $H_{\mathrm{O}} \rtimes H$ is isomorphic to $H$ by the projection $\tau: H_{\mathrm{o}} \rtimes H \rightarrow H$. Furthermore, if ${ }_{H} G \in$ Act and $\pi^{\prime}: G \rightarrow H_{\mathrm{O}}$ is the bundle projection, then $\pi^{\prime}$ preserves the action by (A1), and the map $\pi^{\prime} \rtimes 1_{H}$ corresponds to the projection $\psi: G \rtimes H \rightarrow H$ under the isomorphism.

\subsection{The derived ordered groupoid}

To any ordered functor, we proceed to associate an ordered groupoid which will play a role in our theory similar to that played by the kernel in group theory. This construction first appeared for categories in the work of Quillen [18] and has been rediscovered several times since $[\mathbf{1 5}, \mathbf{2 1}]$. Let $\varphi: G \rightarrow H$ be a morphism of ordered groupoids. Define the derived ordered groupoid of $\varphi$ to be, as a partially ordered set,

$$
\operatorname{Der}(\varphi)=H \times_{\boldsymbol{r}, \boldsymbol{d} \varphi} G=\{(h, g) \mid h \boldsymbol{r}=g \boldsymbol{d} \varphi\} .
$$

Note that if $G$ and $H$ are finite, then $\operatorname{Der}(\varphi)$ is also finite. We define a partial product by $\exists(h, g)\left(h^{\prime}, g^{\prime}\right)$ if $h^{\prime}=h g \varphi$ and $\exists g g^{\prime}$. In this case,

$$
(h, g)\left(h^{\prime}, g^{\prime}\right)=\left(h, g g^{\prime}\right) .
$$


Proposition 3.6. One has that $\operatorname{Der}(\varphi)$ is an ordered groupoid.

Proof. It is straightforward to show that

$$
\operatorname{Der}(\varphi)_{\mathrm{o}}=H \times_{\boldsymbol{r}, \varphi} G_{\mathrm{o}}
$$

and that the equations $(h, g) \boldsymbol{d}=(h, g \boldsymbol{d})$ and $(h, g) \boldsymbol{r}=(h g \varphi, g \boldsymbol{r})$ hold. Then to see that $\operatorname{Der}(\varphi)$ is a category, one performs a routine calculation to check associativity. It is, in fact, a groupoid with $(h, g)^{-1}=\left(h g \varphi, g^{-1}\right)$; for details we refer the reader to [20].

We now show that $\operatorname{Der}(\varphi)$ is an ordered groupoid. First note that if $(h, g) \leqslant\left(h^{\prime}, g^{\prime}\right)$, then $h \leqslant h^{\prime}, g^{-1} \leqslant\left(g^{\prime}\right)^{-1}$, and $g \varphi \leqslant g^{\prime} \varphi$, so

$$
(h, g)^{-1}=\left(h g \varphi, g^{-1}\right) \leqslant\left(h^{\prime} g^{\prime} \varphi,\left(g^{\prime}\right)^{-1}\right)=\left(h^{\prime}, g^{\prime}\right)^{-1} .
$$

If $(h, g) \leqslant(l, k),(r, s) \leqslant(u, v)$ and $\exists(h, g)(r, s), \exists(l, k)(u, v)$, then

$$
(h, g)(r, s)=(h, g s) \leqslant(l, k v)=(l, k)(u, v) .
$$

To see that $\operatorname{Der}(\varphi)_{\mathrm{o}}$ is an order ideal, note that if $(h, g) \leqslant\left(h^{\prime}, e\right)$ with $e \in G_{\mathrm{o}}$, then $g \leqslant e$ so $g \in G_{\mathrm{o}}$ and hence $(h, g) \in \operatorname{Der}(\varphi)_{\mathrm{o}}$. Finally, suppose $(h, e) \leqslant\left(h^{\prime}, g^{\prime}\right) \boldsymbol{d}$ with $e \in G_{\mathrm{o}}$. Then we claim $\left(h,\left(e \mid g^{\prime}\right)\right) \in \operatorname{Der}(\varphi)$. Indeed $h \boldsymbol{r}=e \varphi=\left(e \mid g^{\prime}\right) \boldsymbol{d} \varphi$. But $\left(h,\left(e \mid g^{\prime}\right)\right) \leqslant\left(h^{\prime}, g^{\prime}\right)$, so we now see that $\operatorname{Der}(\varphi)$ is an ordered groupoid.

Define an action of $H$ on $\operatorname{Der}(\varphi)$ as follows: let $\pi: \operatorname{Der}(\varphi) \rightarrow H$ be defined by $(h, g) \pi=h \boldsymbol{d}$ and $A: H_{\mathrm{D}} \times_{\boldsymbol{r}, \pi} \operatorname{Der}(\varphi) \rightarrow \operatorname{Der}(\varphi)$ by $\left(h^{\prime},(h, g)\right) A=\left(h^{\prime} h, g\right)$. First note that since $h \leqslant h^{\prime}$ implies $h \boldsymbol{d} \leqslant h^{\prime} \boldsymbol{d}, \pi$ preserves order. To see that $A$ preserves order, if $\left(h^{\prime},(h, g)\right) \leqslant\left(k^{\prime},(k, l)\right)$, then $\left(h^{\prime} h, g\right) \leqslant\left(k^{\prime} k, l\right)$. The remaining verifications that $(\pi, A)$ is an action are straightforward and as in the category setting [20].

Proposition 3.7. The correspondence Der : Morph $\rightarrow$ Act is a functor.

Proof. Let $(\psi, \rho): \varphi \rightarrow \tau$ be an arrow of Morph. Define $\operatorname{Der}(\psi, \rho)=(\rho \times \psi, \rho)$. It is easy to verify that $\rho \times \psi$ is an ordered functor; in fact, it is a functor as per $[\mathbf{2 0}, \S 3.3]$ and preserves order by definition of the product order. The action is preserved since

$$
\left(h^{h^{\prime}}(h, g)\right)(\rho \times \psi)=\left(\left(h^{\prime} h\right) \rho, g \psi\right)=h^{h^{\prime}} \rho(h \rho, g \psi),
$$

as desired. Functoriality is immediate.

An important example is the following: suppose that $G$ is a group, then $\operatorname{Der}\left(1_{G}\right)$ is the Cayley graph of $G$ with generators $G$, viewed as a groupoid via the multiplication rule $\left(g, g_{1}\right)\left(g g_{1}, g_{2}\right)=\left(g, g_{1} g_{2}\right)$.

\subsection{Proof of the adjunction}

Our next goal is to prove that Der is left adjoint to Sd. We first need the following embedding result. 
Proposition 3.8. Let $\varphi: G \rightarrow H$ be an ordered functor. Define a map $\iota: G \rightarrow$ $\operatorname{Der}(\varphi) \rtimes H$ by

$$
g \iota=((g \boldsymbol{d} \varphi, g), g \varphi) .
$$

Then $\iota$ is an order embedding.

Proof. The map is well defined, since $g \varphi \boldsymbol{d}=(g \boldsymbol{d} \varphi, g) \pi$. If $g \leqslant h$, then $((g \boldsymbol{d} \varphi, g), g \varphi) \leqslant$ $((h \boldsymbol{d} \varphi, h), h \varphi)$. It is a functor, for if $\exists g g^{\prime}$, then

$$
\left(g g^{\prime}\right) \iota=\left(\left(\left(g g^{\prime}\right) \boldsymbol{d} \varphi, g g^{\prime}\right),\left(g g^{\prime}\right) \varphi\right)=((g \boldsymbol{d} \varphi, g), g \varphi)\left(\left(g \boldsymbol{r} \varphi, g^{\prime}\right), g^{\prime} \varphi\right)=g \iota g^{\prime} \iota,
$$

since $g \boldsymbol{r}=g^{\prime} \boldsymbol{d}$. We have that $\iota$ is an order embedding since

$$
((g \boldsymbol{d} \varphi, g), g \varphi) \leqslant\left(\left(g^{\prime} \boldsymbol{d} \varphi, g^{\prime}\right), g^{\prime} \varphi\right)
$$

implies $g \leqslant g^{\prime}$.

Thus $G$ can, in fact, be viewed as an ordered subgroupoid of $\operatorname{Der}(\varphi) \rtimes H$ and $\varphi=\iota \psi$, where $\psi: \operatorname{Der}(\varphi) \rtimes H \rightarrow H$ is the projection. In this manner we obtain a morphism $\left(\iota, 1_{H}\right)$ from $\varphi$ to $\psi$. Observe that the function $\tau: \operatorname{Der}(\varphi) \rtimes H \rightarrow G$ given by $\left((h, g), h^{\prime}\right) \tau=g$ is an ordered functor and that $\iota \tau=1_{G}$. Thus $G$ is a retract of $\operatorname{Der}(\varphi) \rtimes H$. Further properties of this embedding will be studied in Theorem 4.9.

Theorem 3.9. Der : Morph $\rightarrow$ Act is left adjoint to Sd : Act $\rightarrow$ Morph.

Proof. This will follow immediately from [20, Theorem 4.4] once we show that the unit and counit of the adjunction from the category setting are ordered functors in the ordered groupoid setting. Proposition 3.8 shows that the unit $\iota$ is an ordered functor. If $\psi: G \rtimes H \rightarrow H$ is the semidirect product projection, then the counit $\rho: \operatorname{Der}(\psi) \rightarrow G$ is defined by $(h, g) \rho={ }^{h} g$. This is immediately verified to preserve order by the definition of a left action.

We then obtain the following from the usual abstract nonsense [13].

Corollary 3.10. Let $\varphi: G \rightarrow H$ be an ordered functor and $\iota: G \rightarrow \operatorname{Der}(\varphi) \rtimes H$ be the order embedding of (3.1). Then $\left(\iota, 1_{H}\right)$ is a universal arrow from $\varphi$ to the semidirect product functor $\mathbf{S d}$.

\section{A menagerie of morphisms}

In this section we investigate various classes of morphisms and how they relate to the derived and semidirect product functors. From this work, we will obtain our factorization theorems. We begin with some definitions.

We call an ordered functor $\varphi: G \rightarrow H$ identity separating if $\left.\varphi\right|_{G_{\circ}}$ is injective. An ordered functor $\varphi$ is called star injective (or, by some authors, an immersion), respectively, star surjective (or, by some authors, a fibration), if, for all $e \in G_{\mathrm{o}},\left.\varphi\right|_{e \boldsymbol{d}^{-1}}$ : $e \boldsymbol{d}^{-1} \rightarrow e \varphi \boldsymbol{d}^{-1}$ is injective, respectively, surjective; it is called a covering morphism if it is both star injective and star surjective. Star-injective inductive functors correspond to 
idempotent-pure morphisms of inverse semigroups. Indeed, it is straightforward to prove the following.

Proposition 4.1. Let $\varphi: G \rightarrow H$ be a morphism of ordered groupoids. Then $\varphi$ is star injective if and only if $H_{\mathrm{o}} \varphi^{-1}=G_{\mathrm{o}}$.

We now exhibit the following important example of a covering morphism.

Theorem 4.2. Let $\varphi: G \rightarrow H$ be an ordered functor. Then the projection $\sigma$ : $\operatorname{Der}(\varphi) \rightarrow G$ given by $(h, g) \sigma=g$ is a covering morphism.

Proof. Clearly $\sigma$ is an ordered functor. Suppose $(h, g) \boldsymbol{d}=\left(h^{\prime}, g\right) \boldsymbol{d}$. Then $h=h^{\prime}$, whence $\sigma$ is star injective. Let $(h, e) \in \operatorname{Der}(\varphi)_{\mathrm{o}}$ and suppose $g \boldsymbol{d}=e=(h, e) \sigma$. Then, since $h \boldsymbol{r}=e \varphi=g \boldsymbol{d} \varphi,(h, g) \in \operatorname{Der}(\varphi)$ and $(h, g) \boldsymbol{d}=(h, e)$. So $\varphi$ is star surjective and hence a covering morphism.

If $G$ is an ordered groupoid and $e, f \in G_{\mathrm{o}}$, we let $G(e, f)=e \boldsymbol{d}^{-1} \cap f \boldsymbol{r}^{-1}$ denote the hom-set of $e$ and $f$. We call $G(e, e)$ the local group at $e$. It is easy to show, and well known, that the local groups at any two elements of the same $\mathcal{D}$-class are isomorphic. If $\varphi: G \rightarrow H$ is a functor, there is an induced map $\varphi_{e, f}: G(e, f) \rightarrow H(e \varphi, f \varphi)$.

An ordered functor $\varphi: G \rightarrow H$ is called faithful if it is injective when restricted to each hom-set, or, equivalently, it is injective when restricted to each local group. Such morphisms, for inductive groupoids, correspond to $\mathcal{H}$-injective homomorphisms of inverse semigroups. Note that any star-injective functor must be faithful. One says that $\varphi$ is full if for each $e, f \in G_{\mathrm{o}}, \varphi_{e, f}$ is surjective or, equivalently, for each $e \in G_{\mathrm{o}}, \varphi_{e, e}$ is surjective. Full maps are necessarily star surjective. The corresponding concept for inverse semigroups is that of an $\mathcal{H}$-surjective morphism.

Observe that if $\varphi: G \rightarrow H$ is an ordered functor and $e \in H_{0}$, then $e \varphi^{-1}$ is an ordered subgroupoid of $G$. If $\mathbf{V}$ is a class of ordered groupoids, we say that $\varphi$ is a $\mathbf{V}$-morphism if, for all $e \in H_{\mathrm{o}}, e \varphi^{-1} \in \mathbf{V}$. For instance, if $\mathbf{P O}$ is the class of partially ordered sets, then Proposition 4.1 says that an ordered functor if star injective if and only if it is a $\mathbf{P O}$-morphism. If $\mathbf{G}$ is the class of groups, then the $\mathbf{G}$-morphisms are just the identityseparating morphisms.

An ordered groupoid is called combinatorial or aperiodic if its local groups are trivial (or, equivalently, each hom-set has at most one element). The class of aperiodic ordered groupoids will be denoted A. Observe that A-morphisms are precisely faithful ordered functors.

We will call an ordered groupoid a Clifford groupoid if it is precisely the union of its local groups; this class will be denoted $\mathbf{C R}$. Note that $\mathbf{P O}=\mathbf{A} \cap \mathbf{C R}$ : star-injective morphisms are precisely faithful CR-morphisms.

An ordered groupoid is called semisimple if distinct $\mathcal{D}$-equivalent elements are incomparable; observe that $(\mathrm{O} 3)$ and $(\mathrm{O} 4)$ imply that semisimplicity is equivalent to asking that distinct $\mathcal{D}$-equivalent identities be incomparable. We will use $\mathbf{S S}$ to denote the class of semisimple ordered groupoids. Notice that the class SS is closed under taking ordered subgroupoids; hence any morphism with semisimple domain is an SS-morphism. Also note that $\mathbf{P O} \subseteq \mathbf{C R} \subseteq \mathbf{S S}$, so any star-injective ordered functor is an SS-morphism. 
We shall call an ordered groupoid quasi-stable if $\mathcal{D}=\mathcal{J}$; this class will be denoted QS. In this case, the preorder $\leqslant \mathcal{J}$ on the set of $\mathcal{D}$-classes is a partial order.

Lemma 4.3. Let $G$ be an ordered groupoid. Then $G \in \mathbf{Q S}$ if and only if $e^{\prime} \leqslant f \leqslant e$ and $e^{\prime} \mathcal{D} e$ implies $e \mathcal{D} f$, where $e, e^{\prime}, f \in G_{\mathrm{o}}$.

Proof. Necessity is clear. For sufficiency, suppose $D_{1}, D_{2}$ are $\mathcal{D}$-classes with $D_{1} \leqslant \mathcal{J}$ $D_{2}$ and $D_{2} \leqslant \mathcal{J} D_{1}$. Let $e \in D_{2} \cap G_{\mathrm{o}}$. Then, by (2.1), there exists $f \in D_{1}$ with $f \leqslant e$ (and hence $f \in G_{\mathrm{o}}$ ). Similarly, there exists $e^{\prime} \in D_{2} \cap G_{\mathrm{o}}$ with $e^{\prime} \leqslant f$. Thus, by assumption, $D_{1}=D_{2}$.

We then have the following immediate corollary.

Corollary 4.4. SS $\subseteq$ QS.

\subsection{Classifying morphisms}

We now characterize various morphisms in terms of their derived ordered groupoids. First a lemma.

Lemma 4.5. Suppose $\varphi: G \rightarrow H$ is an ordered functor and $(h, e) \mathcal{D}\left(h^{\prime}, e^{\prime}\right)$ in $\operatorname{Der}(\varphi)$ with $e, e^{\prime} \in G_{\mathrm{o}}$. Then $h \boldsymbol{d}=h^{\prime} \boldsymbol{d}$. If, in addition, one has that $h \leqslant h^{\prime}$, then $h=h^{\prime}$ and $e \mathcal{D} e^{\prime}$ in $h \boldsymbol{r} \varphi^{-1}$.

Proof. Any arrow from $(h, e)$ to $\left(h^{\prime}, e^{\prime}\right)$ is of the form $(h, g)$ with $g \boldsymbol{d}=e, g \boldsymbol{r}=e^{\prime}$. But then $h^{\prime}=h g \varphi$, whence $h \boldsymbol{d}=h^{\prime} \boldsymbol{d}$. If, in addition, $h \leqslant h^{\prime}$, then $h=h^{\prime}$ by (O3) and so $h=h g \varphi$, whence $g \varphi=h \boldsymbol{r}$ and so $e \mathcal{D} e^{\prime}$ in $h \boldsymbol{r} \varphi^{-1}$.

Theorem 4.6. Let $\varphi: G \rightarrow H$ be an ordered functor. Then

(1) $\varphi$ is faithful if and only if $\operatorname{Der}(\varphi) \in \mathbf{A}$;

(2) $\varphi$ is a CR-morphism if and only if $(f, e) \mathcal{D}\left(f, e^{\prime}\right)$ with $f \in H_{\mathrm{o}}, e, e^{\prime} \in G_{\mathrm{o}}$ implies $e=e^{\prime}$;

(3) $\varphi$ is an SS-morphism if and only if $\operatorname{Der}(\varphi) \in \mathbf{S S}$;

(4) $p$ is a QS-morphism if and only if $\operatorname{Der}(\varphi) \in \mathbf{Q S}$;

(5) $\varphi$ is star injective if and only if $\operatorname{Der}(\varphi) \in \mathbf{A}$ and $(f, e) \mathcal{D}\left(f, e^{\prime}\right)$ with $f \in H_{\mathrm{o}}, e \in G_{\mathrm{o}}$ implies $e=e^{\prime}$;

(6) $\varphi$ is star surjective if and only if every $\mathcal{D}$-class of $\operatorname{Der}(\varphi)$ contains an object of the form $(f, e)$ with $f \in H_{\mathrm{o}}, e, e^{\prime} \in G_{\mathrm{o}}$.

Proof. (1) First suppose $\varphi$ is faithful and $(h, g) \boldsymbol{d}=(h, g) \boldsymbol{r}$. Then $(h, g \boldsymbol{d})=(h g \varphi, g \boldsymbol{r})$, whence $g \varphi \in H_{\mathrm{O}}$ and $g \boldsymbol{d}=g \boldsymbol{r}$. Thus, since $\varphi$ is faithful, $g \in G_{\mathrm{O}}$ and hence $(h, g) \in$ $\operatorname{Der}(\varphi)_{\mathrm{o}}$; so $\operatorname{Der}(\varphi) \in \mathbf{A}$. Conversely, if $\operatorname{Der}(\varphi) \in \mathbf{A}, g \boldsymbol{d}=g \boldsymbol{r}$, and $g \varphi \in H_{\mathrm{o}}$, then $(g \varphi, g) \in \operatorname{Der}(\varphi),(g \varphi, g) \boldsymbol{d}=(g \varphi, g \boldsymbol{d})=(g \varphi, g \boldsymbol{r})=(g \varphi, g) \boldsymbol{r}$. So, by combinatoriality, $(g \varphi, g) \in \operatorname{Der}(\varphi)_{\mathrm{o}}$, whence $g \in G_{\mathrm{o}}$. Thus $\varphi$ is faithful. 
(2) Suppose that $\varphi$ is a CR-morphism and $(f, e),\left(f, e^{\prime}\right)$ are as in the statement. By Lemma 4.5, $e \mathcal{D} e^{\prime}$ in $f \varphi^{-1}$. Since $\varphi$ is a CR-morphism, $e=e^{\prime}$ as desired. For the converse, let $f \in H_{\mathrm{o}}$ and $g \varphi=f$. Then $(f, g \boldsymbol{d}),(f, g \boldsymbol{r}) \in \operatorname{Der}(\varphi)$ and are connected by the arrow $(f, g)$, whence, by assumption, $g \boldsymbol{d}=g \boldsymbol{r}$. It follows that $f \varphi^{-1} \in \mathbf{C R}$.

(3) Let $\varphi$ be an SS-morphism and suppose $(h, e) \mathcal{D}\left(h^{\prime}, e^{\prime}\right)$ are elements of $\operatorname{Der}(\varphi)$ o with $(h, e) \leqslant\left(h^{\prime}, e^{\prime}\right)$. Then $e, e^{\prime} \in G_{\mathrm{o}}$ and so, by Lemma $4.5, h=h^{\prime}$ and $e \mathcal{D} e^{\prime}$ in $h \boldsymbol{r} \varphi^{-1} \in \mathbf{S S}$. Since $e \leqslant e^{\prime}$, we can conclude $e=e^{\prime}$ and so $\operatorname{Der}(\varphi) \in \mathbf{S S}$. For the converse, suppose $f=e \varphi=e^{\prime} \varphi$ with $e, e^{\prime} \in G_{\mathrm{o}}, e \mathcal{D} e^{\prime}$ in $f \varphi^{-1}$, and $e \leqslant e^{\prime}$. Then $(f, e) \leqslant\left(f, e^{\prime}\right)$. Also if $g \in f \varphi^{-1}$ with $g \boldsymbol{d}=e, g \boldsymbol{r}=e^{\prime}$, then $(f, g)$ is an arrow from $(f, e)$ to $\left(f, e^{\prime}\right)$, whence $(f, e)=\left(f, e^{\prime}\right)$ by semisimplicity of $\operatorname{Der}(\varphi)$. Thus $e=e^{\prime}$ as desired.

(4) Suppose $\varphi$ is a QS-morphism and $\left(h^{\prime}, e^{\prime}\right) \leqslant(k, f) \leqslant(h, e)$ in $\operatorname{Der}(\varphi)_{\text {o }}$ with $(h, e) \mathcal{D}\left(h^{\prime}, e^{\prime}\right)$ and $e, e^{\prime}, f \in G_{\mathrm{o}}$. Then, by Lemma $4.5, h^{\prime}=h$ (whence $k=h$ ) and $e^{\prime} \mathcal{D} e$ in $h \boldsymbol{r} \varphi^{-1}$. But $f \varphi=k \boldsymbol{r}=h \boldsymbol{r}$ so, by Lemma 4.3, it now follows that $f \mathcal{D} e$ in $h \boldsymbol{r} \varphi^{-1}$, whence there exists $g \in h \boldsymbol{r} \varphi^{-1}$ such that $g \boldsymbol{d}=e, g \boldsymbol{r}=f$. Moreover, $(h, g)$ is an arrow from $(h, e)$ to $(k, f)$ in $\operatorname{Der}(\varphi)$, so another application of Lemma 4.3 shows $\operatorname{Der}(\varphi) \in$ QS. Conversely, suppose $\operatorname{Der}(\varphi) \in$ QS and suppose $e, e^{\prime}, f \in G_{\mathrm{O}}$ are such that $f^{\prime}=e \varphi=e^{\prime} \varphi=f \varphi, e^{\prime} \leqslant f \leqslant e$, and $e \mathcal{D} e^{\prime}$ in $f^{\prime} \varphi^{-1}$. Then in $\operatorname{Der}(\varphi)$ we have $\left(f^{\prime}, e^{\prime}\right) \leqslant\left(f^{\prime}, f^{\prime}\right) \leqslant\left(f^{\prime}, e\right)$. Also, if $g \in G$ is such that $g \varphi=f^{\prime}, g \boldsymbol{d}=e, g \boldsymbol{r}=e^{\prime}$, then $\left(f^{\prime}, g\right)$ takes $\left(f^{\prime}, e\right)$ to $\left(f^{\prime}, e^{\prime}\right)$ and so $\left(f^{\prime}, e^{\prime}\right) \mathcal{D}\left(f^{\prime}, e\right)$. Thus, by quasi-stability, $\left(f^{\prime}, f\right) \mathcal{D}\left(f^{\prime}, e\right)$, whence, by Lemma $4.5, e \mathcal{D} f$ in $f^{\prime} \varphi^{-1}$ as desired.

(5) Follows from (1) and (2).

(6) Suppose $\varphi$ is star surjective and let $(h, e) \in \operatorname{Der}(\varphi)_{\mathrm{o}}$. Then $h \boldsymbol{r}=e \varphi$. Thus, since $\varphi$ is star surjective, there exists $g \in G$ with $g \boldsymbol{r}=e$ and $g \varphi=h$. Then $\left(h, g^{-1}\right) \in \operatorname{Der}(\varphi)$, $\left(h, g^{-1}\right) \boldsymbol{d}=(h, g \boldsymbol{r})=(h, e)$ and $\left(h, g^{-1}\right) \boldsymbol{r}=\left(h\left(g^{-1}\right) \varphi, g \boldsymbol{d}\right)=(h \boldsymbol{d}, g \boldsymbol{d})$. So every $\mathcal{D}$-class contains an element of the desired form. For the converse, suppose $e \in G_{\mathrm{O}}$ and $e \varphi=h \boldsymbol{r}$. Then $(h, e) \in \operatorname{Der}(\varphi)$ and so, by assumption, there is an element $\left(f, e^{\prime}\right)$ with $f \in H_{\mathrm{o}}$, $e^{\prime} \in G_{\mathrm{O}}$ in the same $\mathcal{D}$-class as $(h, e)$. Hence there is an edge from $\left(f, e^{\prime}\right)$ to $(h, e)$. This edge must be of the form $(f, g)$ with $g \boldsymbol{d}=e^{\prime}, g \boldsymbol{r}=e$ and $h=f g \varphi=g \varphi$. Thus $\varphi$ is star surjective.

\subsection{Semidirect products and morphisms}

Given a morphism $(\varphi, \rho)$ in Act, we want to investigate the relationship between $\varphi$ and $\varphi \rtimes \rho$. First a definition. Lawson [11] defines a special functor $\varphi: G \rightarrow H$ to be an ordered functor satisfying the following two additional properties.

(S1) If $\exists g_{1} \varphi g_{2} \varphi$, then there exist $g_{1}^{\prime}, g_{2}^{\prime} \in G$ with $g_{1} \varphi=g_{1}^{\prime} \varphi, g_{2} \varphi=g_{2}^{\prime} \varphi$ and $\exists g_{1}^{\prime} g_{2}^{\prime}$;

(S2) If $g_{1} \varphi \leqslant g_{2} \varphi$, then there exists $g_{1}^{\prime} \in G$ with $g_{1}^{\prime} \varphi=g_{1} \varphi$ and $g_{1}^{\prime} \leqslant g_{2}$.

This definition is so formulated as to ensure that $G \varphi$ will be an ordered subgroupoid of $H$. It can be shown that any inductive functor is a special functor [11]. Also, if $\varphi: G \rightarrow H$ is a special functor and $G$ is inductive, then $G \varphi$ is also inductive. 
Theorem 4.7. Let $\left(\varphi, 1_{H}\right):{ }_{H} G \rightarrow{ }_{H} K$ be a morphism in Act. Then

(1) $\varphi$ is a $\mathbf{V}$-morphism (for a class $\mathbf{V}$ of ordered groupoids) if and only if $\varphi \rtimes 1_{H}$ is a V-morphism;

(2) $\varphi$ is surjective if and only if $\varphi \rtimes 1_{H}$ is surjective;

(3) $\varphi$ is full if and only if $\varphi \rtimes 1_{H}$ is full;

(4) $\varphi$ is faithful if and only if $\varphi \rtimes 1_{H}$ is faithful;

(5) $\varphi$ is star injective if and only if $\varphi \rtimes 1_{H}$ is star injective;

(6) $\varphi$ is star surjective if and only if $\varphi \rtimes 1_{H}$ is star surjective;

(7) $\varphi$ is a special functor if and only if $\varphi \rtimes 1_{H}$ is a special functor.

In particular, if $H$ acts on $G$ with bundle projection $\pi: G \rightarrow H_{\mathrm{o}}$, then the projection $\psi: G \rtimes H \rightarrow H$ is star surjective. Furthermore, $\psi$ is a covering if and only if $G \in \mathbf{P O}$ and is a $\mathbf{V}$-morphism, for $\mathbf{V}$ any of $\mathbf{A}$, $\mathbf{S S}$ or $\mathbf{C R}$, if and only if $G \in \mathbf{V}$.

Proof. Let $\pi: G \rightarrow H_{\mathrm{o}}$ and $\pi^{\prime}: K \rightarrow H_{\mathrm{o}}$ be the bundle projections.

(1) Let $f \in K_{\mathrm{o}}$. Then $f \mapsto\left(f, f \pi^{\prime}\right)$ is a bijection between $K_{\mathrm{o}}$ and $(K \rtimes H)_{\mathrm{o}}$. Define a morphism $\tau:\left(f, f \pi^{\prime}\right)\left(\varphi \rtimes 1_{H}\right)^{-1} \rightarrow f \varphi^{-1}$ by $\left(g, f \pi^{\prime}\right) \tau=g$. We show that this is an isomorphism. It is clearly an order isomorphism of partially ordered sets (since $g \varphi=f$ implies $\left.g \pi=g \varphi \pi^{\prime}=f \pi^{\prime}\right)$. To see that $\tau$ is a functor, note that $\left(g_{1}, f \pi^{\prime}\right)\left(g_{2}, f \pi^{\prime}\right)=$ $\left(g_{1}{ }^{g_{2} \pi} g_{2}, f \pi^{\prime}\right)=\left(g_{1} g_{2}, f \pi^{\prime}\right)$. It is now clear that $\varphi$ is a $\mathbf{V}$-morphism if and only if $\varphi \rtimes 1_{H}$ is a $\mathbf{V}$-morphism.

(2) Suppose $\varphi$ is surjective. Let $(k, h) \in K \rtimes H$ and suppose $g \varphi=k$. Then since $g \pi=g \varphi \pi^{\prime}=k \pi^{\prime}$ and $h \boldsymbol{d}=k \pi^{\prime}$, we see that $(g, h) \in G \rtimes H$ and $(g, h)\left(\varphi \rtimes 1_{H}\right)=(k, h)$. Conversely, if $k \in K$, then $\left(k, k \pi^{\prime}\right) \in K \rtimes H$ and so is the image of some $\left(g, k \pi^{\prime}\right) \in G \rtimes H$. But then $g \varphi=k$.

(3) Suppose $\varphi$ is full. Let $(e, e \pi),(f, f \pi) \in(G \rtimes H)_{\mathrm{o}}$ and suppose $(k, h)$ is an arrow from $(e \varphi, e \pi)$ to $(f \varphi, f \pi)$. Then $k \boldsymbol{d}=e \varphi$ and ${ }^{h^{-1}} k \boldsymbol{r}=f \varphi$, whence $k \boldsymbol{r}={ }^{h} f \varphi=\left({ }^{h} f\right) \varphi$ (note that $h$ is an arrow from $e \pi$ to $\left.f \pi=f \varphi \pi^{\prime}\right)$. Let $g \in G$ be such that $g \varphi=k$, $g \boldsymbol{d}=e, g \boldsymbol{r}={ }^{h} f$; such $g$ exists by fullness. Then $(g, h) \in G \rtimes H$ has $(g, h) \boldsymbol{d}=(e, e \pi)$ and $(g, h) \boldsymbol{r}=\left(h^{-1}\left({ }^{h} f\right), f \pi\right)=(f, f \pi)$ and maps to $(k, h)$. For the converse, let $k \in K$ be such that $k \boldsymbol{d}=e \varphi, k \boldsymbol{r}=f \varphi$ with $e, f \in G_{\mathrm{O}}$. Then $\left(k, k \pi^{\prime}\right) \in K \rtimes H$ is an arrow from $\left(e \varphi, k \pi^{\prime}\right)$ to $\left(f \varphi, k \pi^{\prime}\right)$, so, by assumption, there exists $\left(g, k \pi^{\prime}\right) \in G \rtimes H$, with $\left(g, k \pi^{\prime}\right) \boldsymbol{d}=\left(e, k \pi^{\prime}\right)$ and $\left(g, k \pi^{\prime}\right) \boldsymbol{r}=\left(f, k \pi^{\prime}\right)$, mapping to $\left(k, k \pi^{\prime}\right)$. But then $g \varphi=k, g \boldsymbol{d}=e$ and $g \boldsymbol{r}=f$ (since $\left.\left(k \pi^{\prime}\right)^{-1} g \boldsymbol{r}=g \boldsymbol{r}\right)$. So $\varphi$ is full.

(4) Follows from (1).

(5) Follows from (1).

(6) Suppose $\varphi$ is star surjective and let $(e, e \pi) \in(G \rtimes H)_{\mathrm{o}}$. Let $(k, h) \in K \rtimes H$ with $(k, h) \boldsymbol{d}=(e \varphi, e \pi)$. Then $k \boldsymbol{d}=e \varphi$ and $h \boldsymbol{d}=e \pi$. So, by assumption, there exists $g \in G$ 
with $g \boldsymbol{d}=e$ and $g \varphi=k$. Then $(g, h) \in G \rtimes H$ with domain $(e, e \pi)$ and maps to $(k, h)$. For the converse, let $e \in G_{\mathrm{o}}$ and $k \in K$ with $k \boldsymbol{d}=e \varphi$. Then $\left(k, e \varphi \pi^{\prime}\right) \in K \rtimes H$ with domain $\left(e, e \varphi \pi^{\prime}\right)\left(\varphi \rtimes 1_{H}\right)$, whence, by assumption, there exists $\left(g, e \varphi \pi^{\prime}\right) \in G \rtimes H$ with image $\left(k, e \varphi \pi^{\prime}\right)$ and $\left(g, e \varphi \pi^{\prime}\right) \boldsymbol{d}=(e, e \pi)$. Hence $g \boldsymbol{d}=e$ and $g \varphi=k$ as desired.

(7) Suppose that $\varphi$ is a special functor and that

$$
\exists\left(g_{1}, h_{1}\right)\left(\varphi \rtimes 1_{H}\right)\left(g_{2}, h_{2}\right)\left(\varphi \rtimes 1_{H}\right) .
$$

Then $\exists\left(g_{1} \varphi\left({ }^{h_{1}} g_{2}\right) \varphi, h_{1} h_{2}\right)$. Since $\varphi$ is a special functor, there exist $g_{1}^{\prime}, g_{2}^{\prime}$ such that $g_{1}^{\prime} \varphi=$ $g_{1} \varphi, g_{2}^{\prime} \varphi=\left({ }^{h_{1}} g_{2}\right) \varphi$ and $\exists g_{1}^{\prime} g_{2}^{\prime}$. Note that

$$
g_{2}^{\prime} \pi=g_{2}^{\prime} \varphi \pi^{\prime}=\left({ }^{h_{1}} g_{2}\right) \varphi \pi^{\prime}={ }^{h_{1}} g_{2} \pi=h_{1} \boldsymbol{d} .
$$

Similarly, $g_{1}^{\prime} \pi=g_{1} \pi$. It then follows that $\exists\left(g_{1}^{\prime}, h_{1}\right)\left(h_{1}^{-1} g_{2}^{\prime}, h_{2}\right)$ in $G \rtimes H$ and that these elements agree, respectively, with $\left(g_{1}, h_{1}\right)$ and $\left(g_{2}, h_{2}\right)$ when mapped under $\varphi \rtimes 1_{H}$.

Suppose $\left(g_{1} \varphi, h_{1}\right) \leqslant\left(g_{2} \varphi, h_{2}\right)$. Then, since $\varphi$ is a special functor, there exists $g_{1}^{\prime} \in G$ with $g_{1}^{\prime} \varphi=g_{1} \varphi$ and $g_{1}^{\prime} \leqslant g_{2}$. Note that $g_{1}^{\prime} \pi=g_{1}^{\prime} \varphi \pi^{\prime}=g_{1} \pi$ so $\left(g_{1}^{\prime}, h_{1}\right) \in G \rtimes H$ and $\left(g_{1}^{\prime}, h_{1}\right) \leqslant\left(g_{2}, h_{2}\right)$. But $\left(g_{1}^{\prime}, h_{1}\right)\left(\varphi \rtimes 1_{H}\right)=\left(g_{1}, h_{1}\right)\left(\varphi \rtimes 1_{H}\right)$. It follows that $\varphi \rtimes 1_{H}$ is a special functor.

For the converse, suppose first that $\exists g \varphi g^{\prime} \varphi$. Then $g \varphi \pi^{\prime}=g^{\prime} \varphi \pi^{\prime}$ so $g \pi=g^{\prime} \pi$. Consider $(g, g \pi),\left(g^{\prime}, g^{\prime} \pi\right) \in G \rtimes H$. Then $\exists(g \varphi, g \pi)\left(g^{\prime} \varphi, g^{\prime} \pi\right)$ and so, by assumption, there exist $\left(g_{1}, h_{1}\right),\left(g_{2}, h_{2}\right) \in G \rtimes H$ which are composable and map, respectively, to $(g \varphi, g \pi)$, $\left(g^{\prime} \varphi, g^{\prime} \pi\right)$, whence $h_{1}=g \pi=g^{\prime} \pi=h_{2}$. Thus ${ }^{h_{1}} g_{2}=g_{2}$ and so $\exists g_{1} g_{2}$.

Suppose $g \varphi \leqslant g^{\prime} \varphi$. Then $(g \varphi, g \pi) \leqslant\left(g^{\prime} \varphi, g^{\prime} \pi\right)$ in $K \rtimes H$, so there exists $(r, s) \in G \rtimes H$ with $(g \varphi, g \pi)=(r \varphi, s)$ and $(r, s) \leqslant\left(g^{\prime}, g^{\prime} \pi\right)$. So $r \leqslant g^{\prime}$ and $r \varphi=g \varphi$.

To prove the final set of assertions, recall from Example 3.5 that the projection $\psi$ : $G \rtimes H \rightarrow H$ is actually the map $\pi \rtimes 1_{H}$ (under suitable identification of $H$ and $H_{\mathrm{o}} \rtimes H$ ). Since the map $\pi: G \rightarrow H_{\mathrm{o}}$ is automatically star surjective $\left(H_{\mathrm{o}}\right.$ being a partially ordered set), the above results show that $\psi=\pi \rtimes 1_{H}$ is star surjective. It is simple to verify that, for $\mathbf{V}$ any of PO, A, SS or CR, $\pi: G \rightarrow H_{\mathrm{o}}$ is a $\mathbf{V}$-morphism if and only if $G \in \mathbf{V}$. This completes the proof since covering morphisms are precisely star surjective PO-morphisms.

It is well known [11] that covering morphisms always arise from semidirect product projections $\psi: X_{\mathrm{D}} \rtimes H \rightarrow H$, where $X_{\mathrm{D}}$ is a partially ordered set. We shall obtain a new proof of this as an immediate consequence of one of our main theorems.

\subsection{Enlargements}

We now wish to investigate a little further the nature of the embedding (3.1) associated to an ordered functor $\varphi: G \rightarrow H$. To do this, we generalize Lawson's notion of an enlargement $[\mathbf{9}, \mathbf{1 1}]$. We call an ordered functor $\varphi: G \rightarrow G^{\prime}$ enlarging if it satisfies (S2) and

(E1) $G_{\mathrm{o}} \varphi$ is an order ideal of $G_{\mathrm{o}}^{\prime}$; 
(E2) $\varphi$ is full;

(E3) if $e \in G_{\mathrm{o}}^{\prime}$, then there exists $f \in G_{0}$ such that $e \mathcal{D} f \varphi$.

It is easy to verify that (E1) and (E2) imply that $G \varphi$ is an order ideal. Note that an identity-separating, enlarging functor is special. If $\varphi$ is enlarging and an order embedding, we say that $\varphi$ is an enlargement and, following Lawson [9], that $G^{\prime}$ is an enlargement of $G$. Observe that a full, surjective, special functor is automatically enlarging.

Proposition 4.8. The class of enlarging functors is closed under composition.

Proof. Let $\varphi: G \rightarrow G^{\prime}$ and $\rho: G^{\prime} \rightarrow H$ be enlarging functors. A composition of full, ordered functors is a full, ordered functor. For (S2), suppose that $g_{1} \varphi \rho \leqslant g_{2} \varphi \rho$. Then, since $\rho$ satisfies (S2), there exists $g^{\prime} \in G^{\prime}$ such that $g^{\prime} \leqslant g_{2} \varphi$ and $g^{\prime} \rho=g_{1} \varphi \rho$. Since $G \varphi$ is an order ideal, $g^{\prime} \in G \varphi$. Using that $\varphi$ satisfies (S2), we conclude that there exists $g_{1}^{\prime}$ with $g_{1}^{\prime} \leqslant g_{2}$ and $g_{1}^{\prime} \varphi=g^{\prime}$, whence $g_{1}^{\prime} \varphi \rho=g_{1} \varphi \rho$, as desired. For (E1), suppose $h \leqslant e \varphi \rho$ with $e \in G_{\mathrm{o}}$. Then $e \varphi \in G_{\mathrm{o}} \varphi \subseteq G_{\mathrm{o}}^{\prime}$ and so, since $\rho$ is enlarging, $h \in G_{\mathrm{o}}^{\prime} \rho$. Hence there exists $f \in G_{\mathrm{o}}^{\prime}$ with $f \rho=h$. Since $\rho$ satisfies (S2), there exists $g^{\prime} \in G^{\prime}$ with $g^{\prime} \rho=f \rho=h$ and $g^{\prime} \leqslant e \varphi$. But, since $\varphi$ is enlarging, $g^{\prime} \in G_{0} \varphi$, whence $h \in G_{0} \varphi \rho$. For (E3), suppose $e \in H_{\mathrm{o}}$. Then, since $\rho$ is enlarging, there exists $e^{\prime} \in G_{\mathrm{o}}^{\prime}$ such that $e \mathcal{D} e^{\prime} \rho$. Since $\varphi$ is enlarging, there exists $f \in G_{\mathrm{O}}$ such that $e^{\prime} \mathcal{D} f \varphi$, whence $e \mathcal{D} e^{\prime} \rho \mathcal{D} f \varphi \rho$. It follows $\varphi \rho$ is enlarging.

Theorem 4.9. Let $\varphi: G \rightarrow H$ be an ordered functor. Then $\iota: G \rightarrow \operatorname{Der}(\varphi) \rtimes H$, as per (3.1), is an enlargement.

Proof. We already saw in Proposition 3.8 that $\iota$ is an order embedding. To show that (E1) holds, let $e \in G_{0}$. Then $e \iota=((e \varphi, e), e \varphi)$. Suppose $\left((h, g), h^{\prime}\right) \leqslant((e \varphi, e), e \varphi)$. Then $h \boldsymbol{r}=g \boldsymbol{d} \varphi$ and $h^{\prime} \boldsymbol{d}=h \boldsymbol{d}$. Now $g \leqslant e$ implies $g \in G_{\mathrm{o}}$. Hence $g \boldsymbol{d}=g$. Also $h^{\prime} \leqslant e \varphi$ implies $h^{\prime} \in H_{\mathrm{o}}$. So $h^{\prime}=h^{\prime} \boldsymbol{d}=h \boldsymbol{d}$. Now $h \leqslant e \varphi$ so $h \in H_{\mathrm{o}}$. Thus $h=h \boldsymbol{r}=h \boldsymbol{d}=h^{\prime}$. But $h \boldsymbol{r}=g \boldsymbol{d} \varphi=g \varphi$. So we see that $\left((h, g), h^{\prime}\right)=((g \varphi, g), g \varphi)=g \iota$ with $g \in G_{\mathrm{o}}$.

To verify (E2), suppose that $\left((h, g), h^{\prime}\right) \in \operatorname{Der}(\varphi) \rtimes H$ with $\left((h, g), h^{\prime}\right) \boldsymbol{d}=e \iota$ and $\left((h, g), h^{\prime}\right) \boldsymbol{r}=f \iota$ with $e, f \in G_{\mathrm{o}}$. Then first note that $h \boldsymbol{r}=g \boldsymbol{d} \varphi$ and $h \boldsymbol{d}=h^{\prime} \boldsymbol{d}$. Now

$$
\left((h, g), h^{\prime}\right) \boldsymbol{d}=\left((h, g) \boldsymbol{d}, h^{\prime} \boldsymbol{d}\right)=\left((h, g \boldsymbol{d}), h^{\prime} \boldsymbol{d}\right) .
$$

So $e \varphi=h^{\prime} \boldsymbol{d}, g \boldsymbol{d}=e$ and $h=e \varphi$. But

$$
\begin{aligned}
\left((h, g), h^{\prime}\right) \boldsymbol{r} & =\left({ }^{\left(h^{\prime}\right)^{-1}}(h, g) \boldsymbol{r}, h^{\prime} \boldsymbol{r}\right) \\
& =\left({ }^{\left(h^{\prime}\right)^{-1}}(h g \varphi, g \boldsymbol{r}), h^{\prime} \boldsymbol{r}\right) \\
& =\left(\left(\left(h^{\prime}\right)^{-1} h g \varphi, g \boldsymbol{r}\right), h^{\prime} \boldsymbol{r}\right) .
\end{aligned}
$$

So $\left(h^{\prime}\right)^{-1} h g \varphi=f \varphi, g \boldsymbol{r}=f, h^{\prime} \boldsymbol{r}=f \varphi$. Hence $\left((h, g), h^{\prime}\right)=\left((e \varphi, g), h^{\prime}\right)=\left((g \boldsymbol{d} \varphi, g), h^{\prime}\right)$. But $h^{\prime}=h^{\prime} f \varphi=h g \varphi=(e g) \varphi=g \varphi$. Thus $\left((h, g), h^{\prime}\right)=((g \boldsymbol{d} \varphi, g), g \varphi)=g \iota$. So $\iota$ is full. 
Finally, for (E3), let $((h, e), f) \in(\operatorname{Der}(\varphi) \rtimes H)_{\mathrm{o}}$. Then $f \in H_{\mathrm{o}}, e \in G_{\mathrm{o}}, f=h \boldsymbol{d}$, and $e \varphi=h \boldsymbol{r}$. But $\left((h \boldsymbol{r}, e), h^{-1}\right) \in \operatorname{Der}(\varphi) \rtimes H$ since $e \varphi=h \boldsymbol{r}$ and $h^{-1} \boldsymbol{d}=h \boldsymbol{r}$. Now $\left((h \boldsymbol{r}, e), h^{-1}\right) \boldsymbol{d}=((h \boldsymbol{r}, e), h \boldsymbol{r})=((e \varphi, e), e \varphi)=e \iota$, while

$$
\left((h \boldsymbol{r}, e), h^{-1}\right) \boldsymbol{r}=\left({ }^{h}(h \boldsymbol{r}, e) \boldsymbol{r}, h \boldsymbol{d}\right)=\left({ }^{h}(h \boldsymbol{r} e \varphi, e), f\right)=((h, e), f) .
$$

Hence $((h, e), f) \mathcal{D} e \iota$.

Thus we see that $\iota$ is an enlargement.

\section{Factorization theorems}

This section contains the various factorization theorems which form our main body of results. The following two results follow directly from what we have already proved.

Theorem 5.1 (Fibration Theorem). Let $\varphi: G \rightarrow H$ be an ordered functor. Then $\varphi=\iota \psi$, where $\iota$ is an enlargement (with a right inverse) and $\psi$ is an ordered fibration. If $G$ and $H$ are finite, then the domain of $\psi$ can be taken to be finite.

Proof. Let $\iota: G \rightarrow \operatorname{Der}(\varphi) \rtimes H$ be as per (3.1) and let $\psi$ be the semidirect product projection. Then $\iota$ is an enlargement by Theorem 4.9 and $\psi$ is star surjective (a fibration) by Theorem 4.7. Also, we have already shown that there was a retraction from $\operatorname{Der}(\varphi) \rtimes H$ to $G$.

We observe that in the above theorem if $\varphi$ is a $\mathbf{V}$-morphism, where $\mathbf{V}$ is any one of A, SS or QS, then, by Theorems 4.6 and 4.7, we can conclude that $\psi$ is a $\mathbf{V}$-morphism. The following theorem is a more detailed version of the above one.

Theorem 5.2. Let $\varphi: G \rightarrow H$ be an ordered functor. Then there is an enlargement $\iota: G \rightarrow G^{\prime} \rtimes H$ (with a right inverse) such that $\varphi=\iota \psi$ with $\psi$ the projection, and which is universal amongst ordered functors from $G$ into such semidirect products. If $G$ and $H$ are finite, $G^{\prime}$ can be taken to be finite.

Proof. Again let $\iota$ be as per (3.1). The theorem then follows from Theorem 4.9 and Corollary 3.10 .

In the above theorem, if $\varphi$ is a $\mathbf{V}$-morphism and $\mathbf{V}$ is any of $\mathbf{A}, \mathbf{S S}$ or $\mathbf{Q S}$, then $G^{\prime}$ can be chosen to be in $\mathbf{V}$. In particular, the case of an $\mathbf{A}$-morphism can be viewed as the ordered groupoid analogue of the Fundamental Lemma of Complexity [19]. Also recall that $G^{\prime}$ can be taken to have a covering morphism to $G$ (cf. Theorem 4.2).

We now show that Ehresmann's Maximum Enlargement Theorem [11, Theorem 8.3.3] characterizing star-injective morphisms follows simply from our factorization results and from our study of derived ordered groupoids and semidirect products. Lawson has shown [8] that this theorem lies behind the classical theory of idempotent-pure homomorphisms and prehomomorphisms of inverse semigroups due to O'Carroll $[\mathbf{1 6}, \mathbf{1 7}]$ and McAlister [12] (see [11, Chapter 8, §4]). 
Theorem 5.3 (Maximum Enlargement Theorem). Let $\varphi: G \rightarrow H$ be a starinjective ordered functor. Then there is an enlargement $\eta: G \rightarrow K$, and an ordered covering morphism $\varphi^{\prime}: K \rightarrow H$ such that $\eta \varphi^{\prime}=\varphi$.

Lawson showed that such a $K$ has a universal property which characterizes it up to isomorphism as the unique such enlargement [11, Theorem 8.3.5]. This theorem will fall out of a more general result; furthermore, we will have an explicit construction of $K$.

We will need the following straightforward lemma [11, Lemma 8.1.2].

Lemma 5.4. Let $\varphi: G \rightarrow H$ be a star-injective morphism of ordered groupoids. Then, for $x, y \in G, x \leqslant y$ if and only if $x \boldsymbol{d} \leqslant y \boldsymbol{d}$ and $x \varphi \leqslant y \varphi$.

Fix an ordered functor $\varphi: G \rightarrow H$. We let $\mathcal{D}(\varphi)$ denote the set of $\mathcal{D}$-classes of $\operatorname{Der}(\varphi)$. We consider this set with the preorder $\leqslant \mathcal{J}$.

Lemma 5.5. Suppose $H$ acts on the left of an ordered groupoid $G \in \mathbf{Q S}$. Let $\mathcal{D}(G)$ denote the set of $\mathcal{D}$-classes of $G$ ordered by $\leqslant \mathcal{J}$ and let $\rho: G \rightarrow \mathcal{D}(G)$ be the quotient map. Then the action of $H$ on $G$ induces an action of $H$ on $\mathcal{D}(G)$ such that $\left(\rho, 1_{H}\right)$ is an arrow of Act. Furthermore, $\rho$ is a full, surjective, special functor.

Proof. Clearly $\rho$ is a functor since composable arrows are in the same $\mathcal{D}$-class. If, for $g, g^{\prime} \in G, g \leqslant g^{\prime}$, then $g \leqslant \mathcal{J} g^{\prime}$, whence $D_{g} \leqslant \mathcal{J} D_{g^{\prime}}$. Thus $\rho$ is an ordered functor. Suppose $\pi: G \rightarrow H_{\mathrm{o}}$ is the bundle projection. Define $\pi^{\prime}: \mathcal{D}(G) \rightarrow H_{\mathrm{o}}$ by $D_{g} \pi^{\prime}=g \pi$. This is well defined since the image under $\pi$ must be constant on $\mathcal{D}$-classes. Define ${ }^{h} D_{g}=D_{h_{g}}$. Since $D_{g} \pi^{\prime}=g \pi$, the left-hand side is defined if and only if the right-hand side is defined. Also, this definition is independent of the choice of $g$ by Proposition 3.1(6). It is now straightforward to see that this is an action whose very definition ensures that $\left(\rho, 1_{H}\right)$ is an arrow of Act.

To see that $\rho$ is a special functor, observe that $g_{1} \rho$ and $g_{2} \rho$ are composable if and only if $D_{g_{1}}=D_{g_{2}}$. But then $g_{1} \boldsymbol{r} \rho=g_{2} \rho$ and $\exists g_{1} g_{1} \boldsymbol{r}$. If $D_{g_{1}} \leqslant \mathcal{J} D_{g_{2}}$, then, by (2.1), there exists $g \in D_{g_{1}}$ with $g \leqslant g_{2}$. But then $g \rho=g_{1} \rho$. So $\rho$ is a special functor. That $\rho$ is full and surjective is clear.

Now for our main result.

Theorem 5.6. Let $\varphi: G \rightarrow H$ be a QS-morphism. Then $\varphi=\eta \varphi^{\prime}$, where $\eta$ is an enlarging functor and $\varphi^{\prime}$ is an ordered covering morphism. If $G$ and $H$ are finite, the domain of $\varphi^{\prime}$ can be taken to be finite.

Furthermore, if $\varphi$ is star injective, $\eta$ can be taken to be an enlargement (giving Theorem 5.3); if $\varphi$ is star surjective, $\eta$ can be taken to be surjective; in particular, if $\varphi$ is a covering, we can take $\eta$ to be an isomorphism; if $\varphi$ is a CR-morphism, $\eta$ can be taken to be identity separating.

Proof. Since $\varphi$ is a QS-morphism, by Theorem 4.6, $\operatorname{Der}(\varphi) \in$ QS. Hence $\mathcal{D}(\varphi)$ is partially ordered by $\leqslant \mathcal{J}$ and, by Lemma 5.5 , the quotient morphism $\rho: \operatorname{Der}(\varphi) \rightarrow \mathcal{D}(\varphi)$ is a full, surjective, special functor which preserves the $H$-action. Let $\iota: G \rightarrow \operatorname{Der}(\varphi) \rtimes H$ 
be the enlargement of Theorem 4.9 and $\varphi^{\prime}: \mathcal{D}(\varphi) \rtimes H \rightarrow H$ be the projection. Note that $\varphi^{\prime}$ is a covering morphism by Theorem 4.7 .

The induced map $\rho \rtimes 1_{H}: \operatorname{Der}(\varphi) \rtimes H \rightarrow \mathcal{D}(\varphi) \rtimes H$ is a full, surjective special functor by Theorem 4.7 and hence enlarging. Thus, by Proposition $4.8, \eta=\iota\left(\rho \rtimes 1_{H}\right)$ : $G \rightarrow \mathcal{D}(\varphi) \rtimes H$ is an enlarging functor. Also, $\varphi=\eta \varphi^{\prime}$ and so we have proved the first statement.

Suppose $\varphi$ is star injective. We show that $\eta$ is an order embedding. Indeed, if

$$
g \eta=\left(D_{(g \boldsymbol{d} \varphi, g \boldsymbol{d})}, g \varphi\right) \leqslant\left(D_{\left(g^{\prime} \boldsymbol{d} \varphi, g^{\prime} \boldsymbol{d}\right)}, g^{\prime} \varphi\right)=g^{\prime} \eta .
$$

Then $g \varphi \leqslant g^{\prime} \varphi$ and $D_{(g \boldsymbol{d} \varphi, g \boldsymbol{d})} \leqslant \mathcal{J} D_{\left(g^{\prime} \boldsymbol{d} \varphi, g^{\prime} \boldsymbol{d}\right)}$. Hence there exists $(h, e) \in D_{(g \boldsymbol{d} \varphi, g \boldsymbol{d})}$ such that $(h, e) \leqslant\left(g^{\prime} \boldsymbol{d} \varphi, g^{\prime} \boldsymbol{d}\right)$ (whence $h \in H_{\mathrm{o}}, e \in G_{\mathrm{o}}$ ). By Lemma $4.5, h=h \boldsymbol{d}=g \boldsymbol{d} \varphi$ so $e=g \boldsymbol{d}$ by Theorem 4.6 . Thus $g \boldsymbol{d} \leqslant g^{\prime} \boldsymbol{d}$, whence, by Lemma $5.4, g \leqslant g^{\prime}$. It follows that $\eta$ is an order embedding.

Suppose $\varphi$ is star surjective. Since $\eta$ is full, to show that $\eta$ is surjective, it suffices to show that $(\mathcal{D}(\varphi) \rtimes H)_{\mathrm{o}} \subseteq G \eta$. So let $\left(D_{(h, g)}, f^{\prime}\right) \in(\mathcal{D}(\varphi) \rtimes H)_{\mathrm{o}}$; then $f^{\prime} \in H_{\mathrm{o}}$. By Theorem 4.6, there is an element $(f, e) \in D_{(h, g)}$, where $e \in G_{\mathrm{o}}$ and $f \in H_{\mathrm{o}}$. We must then have $f=f \boldsymbol{r}=e \varphi \boldsymbol{d}=e \varphi$. Also, if $\pi^{\prime}: \mathcal{D}(\varphi) \rightarrow H_{\mathrm{o}}$ is the projection, then $D_{(f, e)} \pi^{\prime}=f$, whence $f^{\prime}=f^{\prime} \boldsymbol{d}=D_{(h, g)} \pi^{\prime}=f$. So $\left(D_{(h, g)}, f^{\prime}\right)=\left(D_{(e \varphi, e)}, e \varphi\right)=e \eta$, whence $\eta$ is surjective.

Combining the star-injective and star-surjective cases, we see that $\eta$ is an ordered isomorphism if $\varphi$ is a covering morphism.

Suppose that $\varphi$ is a CR-map and $e, e^{\prime} \in G_{\mathrm{o}}$ with $e \eta=e^{\prime} \eta$. Then $\left(D_{(e \varphi, e)}, e \varphi\right)=$ $\left(D_{\left(e^{\prime} \varphi, e^{\prime}\right)}, e^{\prime} \varphi\right)$ when $e \varphi=e^{\prime} \varphi$ and so $(e \varphi, e) \mathcal{D}\left(e \varphi, e^{\prime}\right)$. By Theorem 4.6, it follows that $e=e^{\prime}$ and so $\eta$ is identity separating.

Immediate corollary results of the theorem and its proof are Theorem 5.3 and the following.

Corollary 5.7. Let $\varphi: G \rightarrow H$ be an ordered functor. Then $\varphi$ is a covering morphism if and only if there is an isomorphism $\tau: G \rightarrow X_{\mathrm{D}} \rtimes H$ such that $X_{\mathrm{D}}$ is a partially ordered set and $\varphi=\tau \psi$, where $\psi$ is the semidirect product projection.

Keeping this corollary in mind, we see that our factorization is as good as can be.

Theorem 5.8. Let $\varphi: G \rightarrow H$ be a QS-morphism and suppose that $\varphi=\tau \psi$, where $\psi$ is an ordered covering morphism which we assume, by the above corollary, to be a semidirect product projection $\psi: X_{\mathrm{D}} \rtimes H \rightarrow H$ ( $X_{\mathrm{D}}$ a partially ordered set). Then there is a unique morphism $\left(\beta, 1_{H}\right):{ }_{H} \mathcal{D}(\varphi) \rightarrow{ }_{H} X_{\mathrm{D}}$ in Act such that $\tau=$ $\eta\left(\beta \rtimes 1_{H}\right)$.

Proof. By Corollary 3.10, there is a unique morphism $\left(\alpha, 1_{H}\right):{ }_{H} \operatorname{Der}(\varphi) \rightarrow{ }_{H} X_{\mathrm{D}}$ in Act such that $\tau=\iota\left(\alpha \rtimes 1_{H}\right)$. Since $X_{\mathrm{D}}$ is a partially ordered set, $\alpha$ factors through the projection $\rho: \operatorname{Der}(\varphi) \rightarrow \mathcal{D}(\varphi)$ as $\rho \beta$, where $\beta$ preserves the action. Also

$$
\eta\left(\beta \rtimes 1_{H}\right)=\iota\left(\rho \rtimes 1_{H}\right)\left(\beta \rtimes 1_{H}\right)=\tau .
$$


We are thus left with checking uniqueness. If $\beta^{\prime}$ is another such morphism, then $\rho \beta^{\prime}=\alpha$ by uniqueness of $\alpha$. Since $\rho$ is surjective, it follows that $\beta^{\prime}=\beta$.

The proof of this theorem motivated the consideration of $\mathcal{D}(\varphi)$ in the proof of Theorem 5.6.

Acknowledgements. The author was supported in part by NSF-NATO postdoctoral fellowship DGE-9972697, by Praxis XXI scholarship BPD, 16306 98, and by FCT through Centro de Matemática da Universidade do Porto. I am greatly indebted to Mark V. Lawson, both for his constant interest, and for his innumerable suggestions on how to improve this work. I also thank Stuart W. Margolis for several interesting email discussions and Bret Tilson, both for his interest in this work, and for the insight he imparted to me while we worked together on $[\mathbf{2 0}]$. Finally, I add a humble thanks to the anonymous referee whose many suggestions did much to ameliorate this paper. I would also like to thank Suzana Mendes for her careful reading of this paper.

\section{References}

1. R. Brown, Groupoids as coefficients, Proc. Lond. Math. Soc. (3) 25 (1972), 413-426.

2. C. Ehresmann, Oeuvres complète et commentées (ed. A. C. Ehresmann), Supplements to Cahiers de Topologie et Géométrie Différentielle, Amiens (1980-1983).

3. G. M. S. Gomes And M. B. Szendrei, Idempotent pure extensions by inverse semigroups via quivers, J. Pure Appl. Algebra 127 (1998), 15-38.

4. A. Grothendieck, Catégories fibrées et descente, in Seminaire de geo-métric algébrique de l'Institut des Hautes Etudes Scientifiques, Paris, 1961.

5. C. H. Houghton, Wreath products of groupoids, J. Lond. Math. Soc. (2) 10 (1985), 179-188.

6. T. B. K. Kientzle, Categorial generalizations of classical monoid theory, $\mathrm{PhD}$ thesis, University of California at Berkeley (1992).

7. M. V. LAWSON, The geometric theory of inverse semigroups, I, E-unitary inverse semigroups, J. Pure Appl. Algebra 67 (1990), 151-177.

8. M. V. LAWSON, The geometric theory of inverse semigroups, II, $E$-unitary inverse semigroups, J. Pure Appl. Algebra 83 (1992), 121-139.

9. M. V. Lawson, Enlargements of regular semigroups, Proc. Edinb. Math. Soc. 39 (1996), 425-460.

10. M. V. Lawson, A class of actions of inverse semigroups, J. Algebra 179 (1996), 570-598.

11. M. V. LaWson, Inverse semigroups (World Scientific, Singapore, 1998).

12. D. B. McAlister, Groups, semilattices and inverse semigroups, II, Trans. Am. Math. Soc. 196 (1974), 231-251.

13. S. MacLane, Categories for the working mathematician (Springer, New York, 1971).

14. S. W. MARGolis and J.-E. Pin, Inverse semigroups and extensions of groups by semilattices, J. Algebra 110 (1987), 277-297.

15. W. R. NiCO, Wreath products and extensions, Houston J. Math. 9 (1983), 71-99.

16. L. O'CARroll, Inverse semigroups as extensions of semilattices, Glasgow Math. J. 16 (1975), 12-21.

17. L. O'CARRoll, Idempotent determined congruences on inverse semigroups, Semigroup Forum 12 (1976), 233-244. 
18. D. Quillen, Higher algebraic K-theory, I, in Algebraic K-theory (ed. H. Bass), Lecture Notes in Mathematics, vol. 341 (Springer, 1973).

19. J. Rhodes, The fundamental lemma of complexity for arbitrary finite semigroups, Bull. Am. Math. Soc. 74 (1968), 227-268.

20. B. Steinberg And B. Tilson, Categories as algebras, II, University of Porto (2000), Preprint.

21. B. Tilson, Categories as algebra, J. Pure Appl. Algebra 48 (1987), 83-198. 